\title{
3-D Printed Radar Absorber with Meta-material Features for X-band Application
}

\author{
${ }^{* 1,2}$ ABDULLAHI, MB; ${ }^{1}$ ALI, MH \\ ${ }^{*}$ Department of Physics, Bayero University Kano Nigeria \\ ${ }^{2}$ Department of Physics, Usmanu Danfodiyo University Sokoto Nigeria \\ *Corresponding authorEmail: abdullahi.bello@udusok.edu.ng, mhali.phy@buk.edu.ng
}

\begin{abstract}
This paper presents a structured radar absorber with metamaterial features based on graphite SLS composite. The unit cell of the proposed design was simulated on COMSOL Multiphysics to determine its frequencydependent absorption characteristics and fabricated using low-cost selective laser sintering 3-D printing technology. The measurement and simulation results showed an effective absorption bandwidth of $1.04 \mathrm{GHz}$ and $2.08 \mathrm{GHz}$ respectively. The optimized structure however, revealed broadband absorption in a frequency range between 8.35 to $12.20 \mathrm{GHz}$ (X band) under normal incidence. Besides, the absorption performance under different polarizations and incident angles were investigated. Results indicated that the absorber exhibits polarization indifference and high absorptivity at a wide angle of incidence. The advantages of low cost, ultra-broad operating band, wide-angle feature, and polarization insensitivity made the proposed absorber a promising candidate in military and civilian applications.
\end{abstract}

\section{DOI: https://dx.doi.org/10.4314/jasem.v25i8.39}

Copyright: Copyright () 2021 Abdullahi and Ali. This is an open access article distributed under the Creative Commons Attribution License (CCL), which permits unrestricted use, distribution, and reproduction in any medium, provided the original work is properly cited.

Dates: Received: 10 May 2021; Revised: 28 June 2021; Accepted: 01 July 2021

Keywords: 3D Printing, Graphite SLS, Metamaterial, Radar, Wide-Angle

As a material designed to absorb incident $\mathrm{RF}$ radiation as effectively as possible from as many likely incident directions, radar absorbing material (RAM) had received significant attention in academia and industry for quite a long time. Radar absorbing materials are specifically used in stealth technology to disguise a vehicle or structure from radar detection (Silva and Kretly 2011). Broadband absorption at ultrathin thickness is an important requirement for the practical application of radar absorbers which proves to be a difficult task because the thickness reduction leads to a decrease of the bandwidth (Abdalla and $\mathrm{Hu} 2012$ ). With the advent of metamaterials, perfect absorption can be achieved at ultrathin thickness as reported in 2008 by Landy and co. (Landy et al. 2008). Meanwhile, several inventions that attempt to broaden the narrowband features of metamaterial absorbers (MMAs) while aiming to meet different requirements and applications have been reported in the last two decades (Y. Fan et al. 2018; Tiep et al. 2018; Pang et al. 2012; H. Luo et al. 2014; Y. Z. Cheng et al. 2012; Rahmanzadeh, Rajabalipanah, and Abdolali 2018; $\mathrm{Kim}$ et al. 2017). The working frequencies of absorbers demonstrated previously spanned from the microwave band (Sood and Tripathi 2017; Y. Cheng et al. 2016; Zhang et al. 2015; S. Fan and Song 2018), through Terahertz band (Tao et al. 2008; Hokmabadi et al. 2013; Zhu et al. 2014; Wang et al. 2014), to the optical band (Hu et al. 2009; Liu et al. 2011). According to (Ren and Yin 2018), low cost and broad operating bandwidth features of MMAs are two pressing issues as they are highly needed in different applications. In this regard, new broadband absorbing materials and methods for low-cost production are receiving a lot of interest (Kjelgard, Wisland, and Lande 2018) among the electromagnetic absorbers research community. Additive manufacturing technology which is also known as 3D printing is a promising processing technology for fabricating structures and devices with different geometries using computer-aided design (Nadgorny and Ameli 2018). It offers high efficiency, convenience, and a low-cost fabrication process that involves printing successive layers of a given material on top of each other. Recently, 3D printing systems have been utilized to manufacture MMAs of different structural designs and material base (Kronberger and Soboll 2016; D. Zhou, Huang, and Du 2016; Wu et al. 2020; Jiang et al. 2018). However, the main hindrance for the fabrication of broadband MMAs using 3D printing technology is the limited range of materials compatible with 3D printers. To address this problem, few reports on dielectric and microwave absorption properties of commercially available 3D printing material have been presented (Kronberger and Soboll 2016; Jiang et al. 2018; Arbaoui et al. 2016). None of these reports though considered graphite SLS 3D printing composite. Therefore, a cross-block structured broadband absorber with metamaterial features for radar absorption at $\mathrm{X}$ band and fabricated using lowcost 3D printing technology is presented in this study. The graphite SLS material is introduced in the design of the absorber for the first time. A preliminary designed structure is 3D printed via selective laser sintering (SLS) technique and its absorption performance measured afterward. Good agreement between measured and simulated results of the preliminary absorber was observed. 


\section{MATERIALS AND METHODS}

Design of the Structure and Simulation: The proposed absorber is a three-layer structure consisting of a crossshaped surface structure, square block middle layer, and conventional single slab bottom layer as revealed in Figure 1. A copper film of $0.04 \mathrm{~mm}$ thickness was used in the design as a ground plane to prevent transmission of the waves beyond the structure. The schematic of the unit cell of the proposed radar absorber with metamaterial features (RAMF) shown in Figure 1 was simulated by using the frequency domain solver of COMSOL Multiphysics based on finite element method (FEM) to investigate its absorption characteristics. Periodic boundary conditions through perfect electric conductor (PEC) boundary on two opposite sides along the $x$-directions and perfect magnetic conductor boundary (PBC) on the other two opposite sides along the $y$-direction which sets tangential fields to 0 are used to mimic an infinite periodic structure (Hui Luo et al. 2019), and a plane wave is incident along $z$-direction with the electric field polarized along the $y$-direction (TE) as the excitation source.
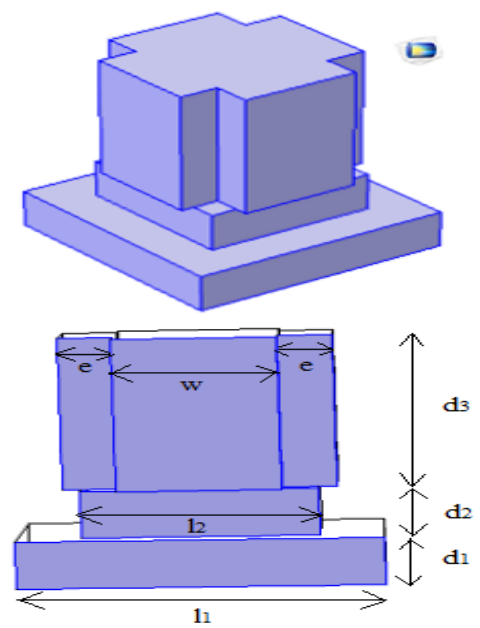

Fig 1: Designed unit cell radar absorbing metamaterial with threelayer cross-block structure (a) model of periodic unit (b) model size. $l_{1}=4.0 \mathrm{~mm}, l_{2}=2.6 \mathrm{~mm}, w=1.8 \mathrm{~mm}, e=0.6 \mathrm{~mm}, d_{1}=$ $0.8 \mathrm{~mm}, d_{2}=0.8 \mathrm{~mm}, d_{3}=2.6 \mathrm{~mm}$

Impedance boundary condition (IBC) which treats any material behind the boundary as being infinitely large was chosen for the ground plane. Physics-controlled tetrahedral meshing was used in the simulation while the output of the simulation software was the scattering parameters ( $s$-parameters).

Fabrication and Measurement: To experimentally investigate the reflectivity performance of the proposed RAMF structure, a model of $180 \times 180 \mathrm{~mm}^{2}$ composed of 45 units cells periodically extended in the $x$ and $y$ directions with a period of $9 \mathrm{~mm}$ was considered for fabrication. It was fabricated using selective laser sintering (SLS) process at a UK based graphite additive manufacturing company facility, and a partially enlarged picture of the $3 \mathrm{D}$ printed RAMF is presented in Figure 2. The material is Graphite SLS, an exclusive material from the company which was obtained from the mixture of a Nylon 12 (PA) powder and a graphite powder. The fabricated absorber structure performance was measured at ABS technics' (Kenis 2020) laboratory to verify the rationality of the COMSOL Multiphysics design. Naval Research Laboratory (NRL) arch measurement procedure was adopted to obtain the absorber reflection loss. The angle of incidence during the measurement is $10^{\circ}$ for each antenna versus the normal on the reflective metal plate for TE polarized incident wave. The measurement result was then compared with the simulated result.

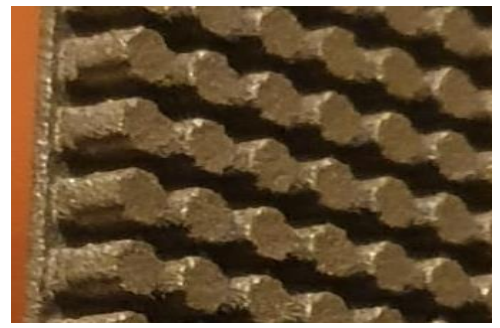

Fig 2: 3D printed radar-absorber with metamaterial features

Geometric Dimension Optimization: The measured and simulated results of the $3 \mathrm{D}$ printed RAMF demonstrate below $-10 \mathrm{~dB}$ reflectivity in the 10 to 12 $\mathrm{GHz}$ range only. To obtain an absorber with below $10 \mathrm{~dB}$ reflectivity encompassing the entire $\mathrm{X}$-band of the radar frequencies, the unit cell geometric dimensions were optimized by using COMSOL Multiphysics' in-built Nelder-Mead method to get the desired frequency range.

\section{RESULTS AND DISCUSSIONS}

The measured reflectivity result of the $3 \mathrm{D}$ printed absorber at an incident angle of $10^{\circ}$ for TE polarized wave is shown in Figure 3. For better comparison, the simulated reflectivity was plotted alongside the measured reflectivity in Figure 4. Comparison of the measurement and simulation results showed reasonable agreement since the effective absorption bandwidth, often defined as the corresponding frequency range in which reflectivity is $\leq-10 \mathrm{~dB}$, was $1.04 \mathrm{GHz}$ for measured and $2.08 \mathrm{GHz}$ for simulated. Deviations between simulated and measured results observed could be credited to the imperfections resulting from the fabrication and measurement processes. The measurement results however, validated the simulation results which enable us to optimize the design in COMSOL Multiphysics to achieve better results. 


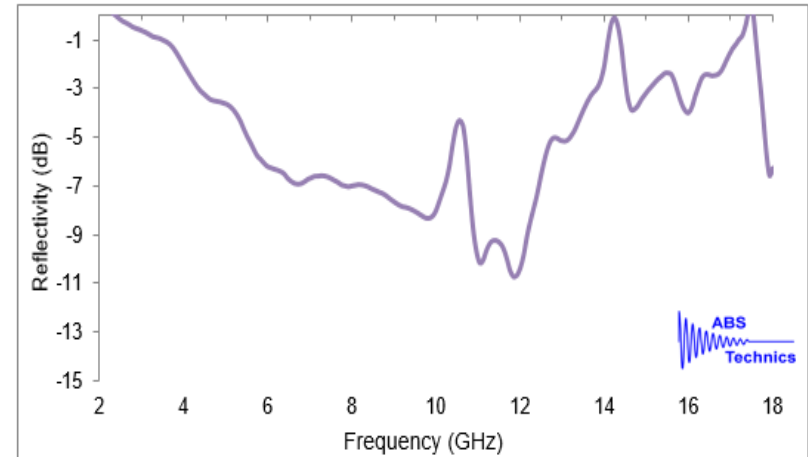

Fig 3: Measured Reflectivity Plots at $10^{\circ}$ Incidence of TE Polarized Wave

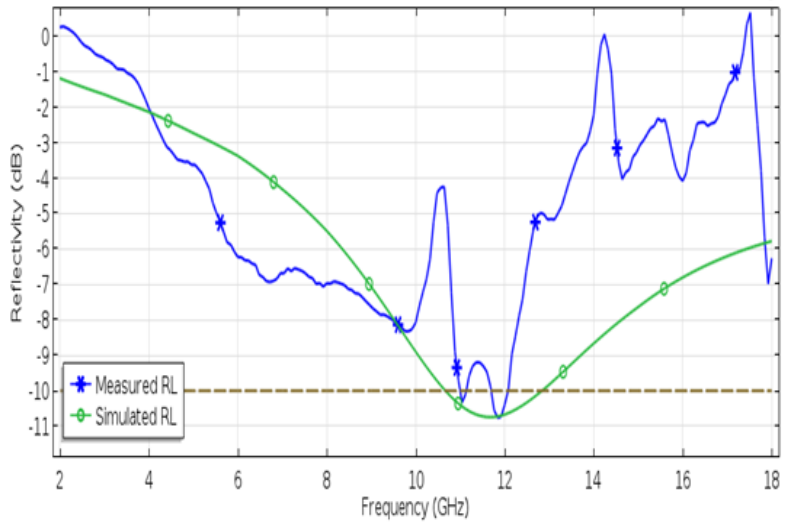

Fig 4: Measured and Simulated Reflectivity Plots at $10^{\circ}$ Incidence of TE Polarized Wave

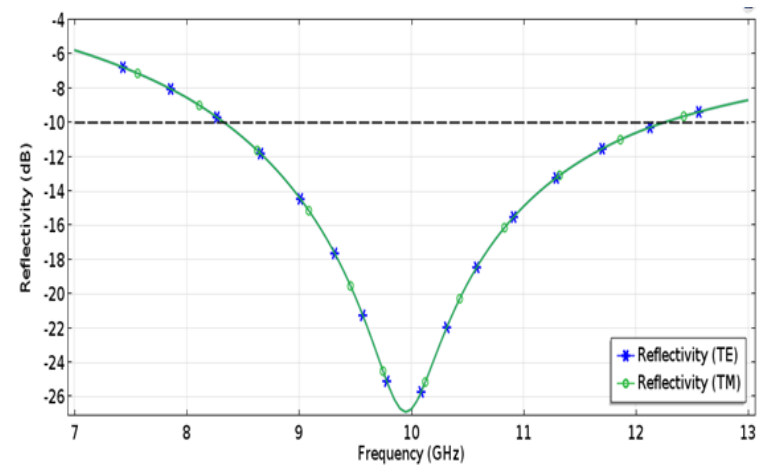

Fig 5: Simulated Reflectivity Plots of the Optimized Structure at Normal Incidence for TE and TM Polarizations.

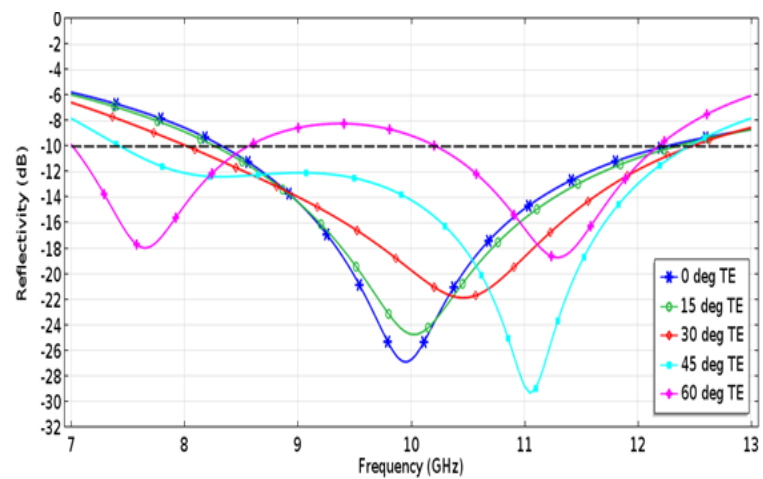

Fig 6: Simulated Reflectivity of the Optimized Structure at Normal and Oblique Incidence for TE Polarized Wave
To get broadband absorption characteristics, the geometric dimensions of the designed absorber are optimized and the optimized dimensions obtained are $l_{1}=$ $8.0 \mathrm{~mm}, l_{2}=4.5 \mathrm{~mm}, w=3.1 \mathrm{~mm}$, $e=1.7 \mathrm{~mm}, \quad d_{1}=0.7 \mathrm{~mm}, d_{2}=$ $1.2 \mathrm{~mm}, d_{3}=1.6 \mathrm{~mm}$. Its simulated reflectivity at normal incidence for both TE and TM polarizations is presented in Figure 5. The effective absorption bandwidth was greatly improved, covering almost the entire frequency range of interest (X band) as depicted in Figure 5 while at the same time achieving decreased absorber thickness. The new optimized absorber thickness is $3.5 \mathrm{~mm}$ only, achieving $0.7 \mathrm{~mm}$ decrease in absorber thickness compared to the printed absorber thickness. The curves for the TE and TM polarizations were observed to overlap at normal incidence. Absorber angle of incidence tolerance is evaluated to assess its suitability for practical application which demands a wide angle of incidence performance. Simulations were carried out for $\mathrm{TE}$ and $\mathrm{TM}$ modes under different angles of incidence varying from $0^{\circ}$ to $60^{\circ}$ at steps of $15^{0}$.

Figure 6 reveals the absorber response under different angles of incidence for TE mode. It can be observed from the figure that as the angle of incidence increased to $45^{\circ}$, the effective bandwidth increased, and absorption peaks shifted to higher frequencies. At $60^{\circ}$ dual reflectivity band was noticed between 2-4 GHz and between 8-12 $\mathrm{GHz}$ in which reflectivity was $\leq-10$ $\mathrm{dB}$. Generally, the absorption level decreases with increasing angle of incidence. For TM polarization, angles of incidence were varied from $0^{\circ}$ to $45^{\circ}$ in steps of $15^{\circ}$ as can be seen in Figure 7. Effective bandwidth similarly increases as the angle of incidence increases while the absorption peaks shifted to lower frequencies. Likewise, absorption level generally decreases with the increase of angle of incidence. 


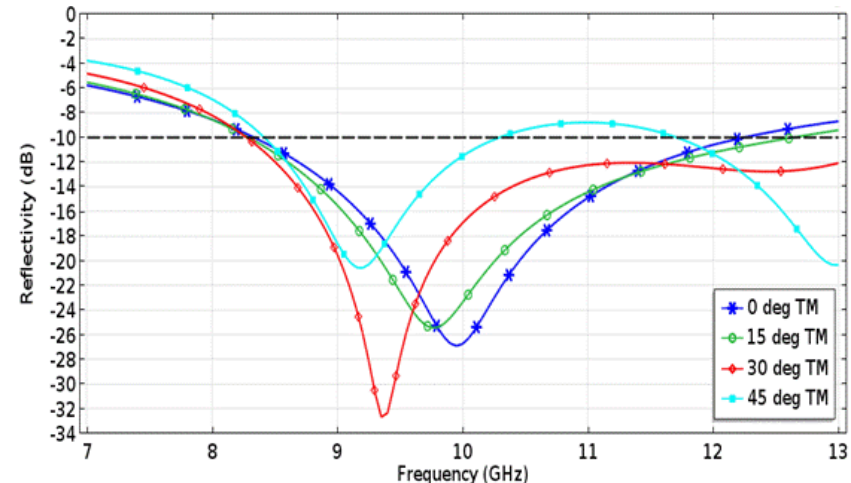

Fig 7: Simulated Reflectivity of the Optimized Structure at Normal and Oblique Incidence for TM Polarized Wave

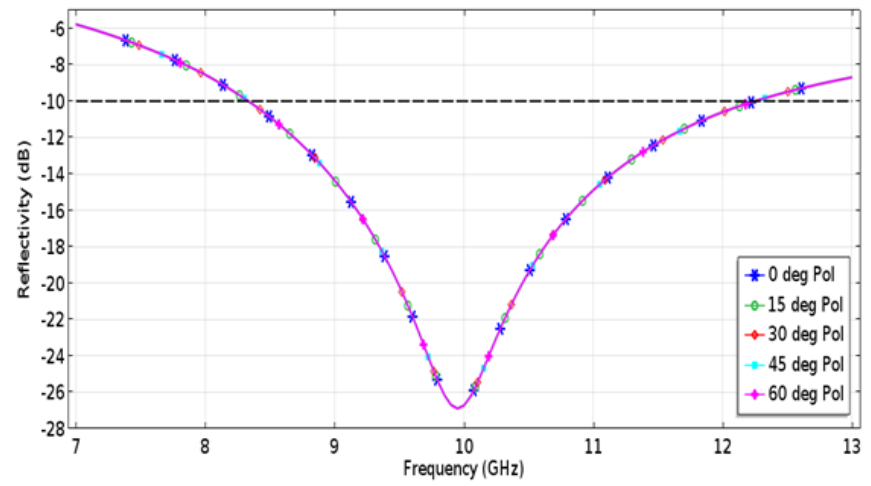

Fig 8: Simulated Reflectivity Plots of Different Polarization Angles at Normal Incidence for Optimized Structure
Another valuable characteristic of absorbers was the ability to respond well in different polarization angles. The present design's response to different polarization angles was investigated for normal incident wave. Simulation result for polarization angles varied from $0^{\circ}$ to $60^{\circ}$ in step of $15^{\circ}$ was shown in Figure 8, which indicated polarization independency characteristics as the curves overlapped over one another due to fourfold symmetry of the absorber unit cell design. Power loss density was simulated at $8.35,10$, and 12.20 $\mathrm{GHz}$ when the incident angle was $0^{\circ}$ to investigate the physical absorption mechanism of the proposed RAMF and results are shown in Figure 9. As depicted in Figure 9a which is for $8.35 \mathrm{GHz}$, power loss was mostly pronounced at the surface of the bottom layer of the structure and side faces of the middle layer along the direction of the electric field component of the incident wave..
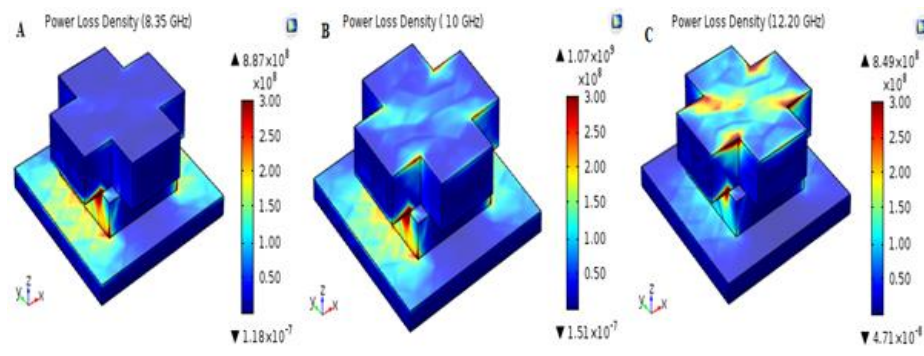

Fig 9: Simulated Power Loss Density Distribution of Graphite SLS based Radar Absorber with Metamaterial Features
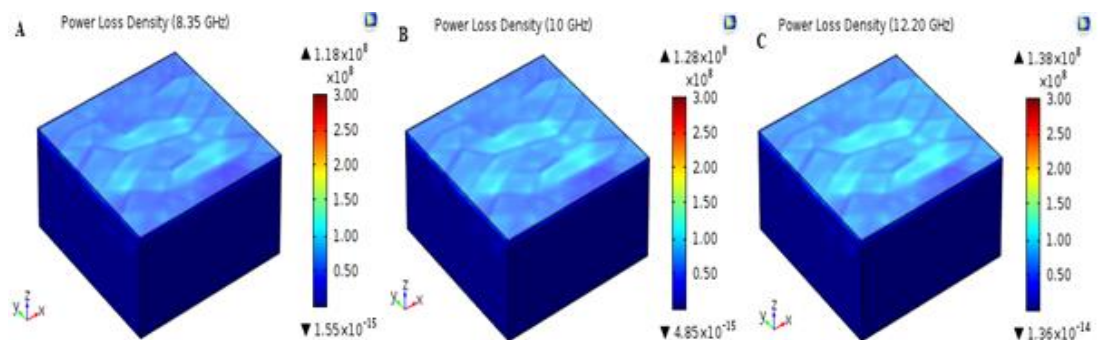

Fig 10: Simulated Power Loss Density Distribution of Graphite SLS based Conventional Absorber

A similar loss pattern was observed at the resonant frequency of $10 \mathrm{GHz}$ as revealed in Figure $9 \mathrm{~b}$ though losses around the four corners located in the middle portion of the cross-shaped surface layer added to it. At this frequency, peak absorption was attained hence the involvement of all the absorber layers for maximum energy absorption. Finally, power loss at $12.20 \mathrm{GHz}$ was observed to be concentrated at the shoulder corners of the cross-shaped surface layer and side faces of the middle layer according to Figure 9c. Thus, we can infer that absorption occurred mainly at the bottom and middle layers at lower frequencies of 
the X-band; at higher frequencies surface and middle layers contributed significantly to energy absorption while at resonant and around the resonant frequencies all the three layers of the proposed structure are actively involved in the energy absorption. Figure 10 illustrates power loss distribution in a conventional absorber made of the same material and thickness as the present RAMF for comparison. For all the frequencies considered, power loss occurred mainly on the surface with a similar loss distribution pattern and a slight increase in peak power loss intensity. The peak power loss densities increased from $1.18 \times 10^{8} \mathrm{Wm}^{-2}$ at $8.35 \mathrm{GHz}$, to $1.28 \times 10^{8} \mathrm{Wm}^{-2}$ at $10 \mathrm{GHz}$, and $1.38 \times 10^{8}$ $\mathrm{Wm}^{-2}$ at $12.20 \mathrm{GHz}$ for the unstructured absorber.

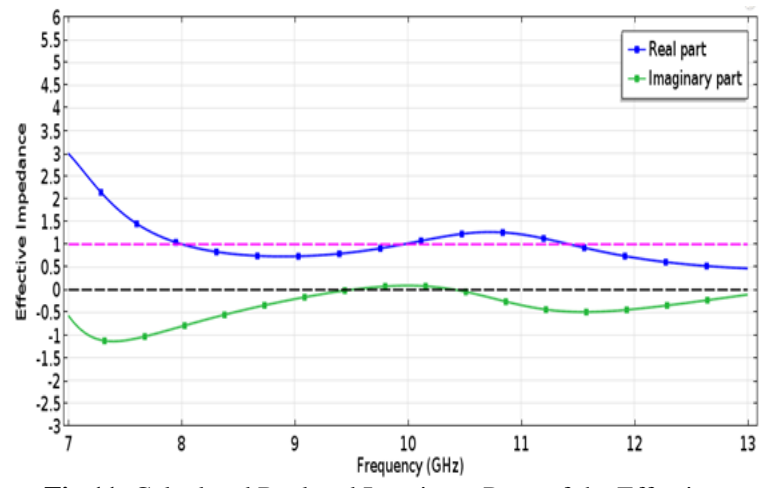

Fig 11: Calculated Real and Imaginary Parts of the Effective Impedance Spectrum of the Proposed RAMF

Therefore, structuring the proposed absorber to its present model gave it extra abilities of electromagnetic waves absorption, so making it behaving as a metamaterial in which its absorption characteristics depend not only on the chemical composition of the graphite SLS material but on its structural design and geometric dimensions. It is, therefore, appropriate to describe the present 3D printed structure as a polarization-independent and wide angle of incidence radar absorber with metamaterial features for $\mathrm{X}$ band applications. For effective and broadband absorption realization in the design of electromagnetic absorbers, effective impedance $\left(Z_{\text {eff }}\right)$ of such absorbers (Q. Zhou et al. 2017) should matched to that of the air. Thus, the effective impedance of the present RAMF was calculated from the simulated complex $\mathrm{S}$ parameters and plotted in Figure 11. As revealed in the plot, the real part of effective impedance of the RAMF was close to unity while its imaginary part was close to zero for all absorption frequencies in the $\mathrm{X}$ band, which resulted in minimized reflections over a wide frequency range thus making it to exhibit broadband absorption property.

Conclusion: In this paper, we have confirmed the feasibility of using graphite SLS material to design and
3D print a metamaterial absorber for X-band application. Simulation results of the optimized RAMF demonstrated broadband, wide angle of incidence and polarization insensitive property for both TE and TM waves. Additionally, the RAMF offered cost-effective solution with exceptional promising attributes, making it suitable for applications in stealth technology and electromagnetic interference shielding.

Acknowledgement: The authors would like to thank the General Manager of ABS Technics, Johan L. Kennis for offering to carry out the measurement of absorption performance of the 3D printed absorber at their facility located in Mol, Belgium at no cost.

\section{REFERENCES}

Abdalla, M. A; Zihrun, Hu (2012). On The Study of Development of $\mathrm{X}$ Band Metamaterial Radar Absorber.Adv. Electromagnetics 1 (3). 3.25.

Arbaoui, Y; Laur, V; Maalouf, A; Quéffélec, P; Passerieux, D; Delias, A; Blondy, P (2016). Full 3-D Printed Microwave Termination: A Simple and Low-Cost Solution. IEEE Transact. Microwave Theo. Techn. 64 (1): $271-78$

Cheng, Y. Z; Wang, Y; Niea, Y; Gong, R. Z; Xiong, X; Wang, X (2012). Design, Fabrication and Measurement of a Broadband Polarization-Insensitive Metamaterial Absorber Based on Lumped Elements. J. Appl. Phys. 111 (4): 044902.

Cheng, Y; He, B; Zhao, J; Gong, R (2016). Ultra-Thin LowFrequency Broadband Microwave Absorber Based on Magnetic Medium and Metamaterial. J. Elect. Mat. 10: 122-131

Fan, S; Song, Y (2018). Bandwidth-Enhanced PolarizationInsensitive Metamaterial Absorber Based on Fractal Structures. Journal of Applied Physics 123 (8): 085110-1 to $085110-6$

Fan, Y; Wang, J; Li, Y; Pang, Y; Zheng, L; Xiang, J; Zhang, J; Qu, S (2018). Ultra-Thin and -Broadband Microwave Magnetic Absorber Enhanced by Phase Gradient Metasurface Incorporation. Journal of Physics D: Applied Physics 51: 215001.

Hokmabadi, M. P; Wilbert, D. S; Kung, P; Kim, S. M (2013). Design and Analysis of Perfect Terahertz Metamaterial Absorber by a Novel Dynamic Circuit Model. OPTICS EXPRESS 21 (14): 16455-16465.

Hu, C; Zhao, Z; Chen, X; Luo, X (2009). Realizing nearPerfect Absorption at Visible Frequencies." OPTICS EXPRESS 17 (13): 11039-11044.

Jiang, W; Yan, L; Ma, H; Fan, Y; Wang, J; Feng, M; Qu S (2018). Electromagnetic Wave Absorption and 
Compressive Behavior of a Three-Dimensional Metamaterial Absorber Based on 3D Printed Honeycomb. Scientific Reports 8: 1-7

Kenis, J. L (2020). ABS Technics. 2020. www.abstechnics.com

Kim, Y. J; Hwang, J. S; Yoo, Y. J; Khuyen, B. X; Rhee, J. Y; Chen, X. F; Lee, Y. P (2017). Ultrathin Microwave Metamaterial Absorber Utilizing Embedded Resistors. J. Phys. D Appl. Phys. 50 (40): 405110.

Kjelgard, K. G; Wisland, D. T; Lande, T. S (2018). 3D Printed Wideband Microwave Absorbers Using Composite Graphite / PLA Filament. In Proceedings of 48th European Microwave Conference, 859-862

Kronberger, R; Soboll, P (2016). 3D-Printed Frequency Selective Surfaces for Microwave Absorbers. In Proceedings of the 46th European Microwave Conference ISAP2016, Okinawa, Japan, 596-599.

Landy, N. I; Sajuyigbe, S; Mock, J. J; Smith, D. R; Padilla, W. J (2008). Perfect Metamaterial Absorber. Physical Review Letters 100 (20): 1-4

Liu, X; Tyler, T; Starr, T; Starr, A. F; Jokerst, N. M; Padilla, W. J (2011). Taming the Blackbody with Infrared Metamaterials as Selective Thermal Emitters. Physical Review Letters 107 (4): 045901.

Luo, H; Hu, X; Qiu, Y; Zhou, P (2014). Design of a WideBand Nearly Perfect Absorber Based on MultiResonance with Square Patch. Solid State Commun. 188: 5-11

Luo, H;, Chen, F; Wang, X; Dai, W; Xiong, Y; Yang, J; Gong, R (2019). A Novel Two-Layer Honeycomb Sandwich Structure Absorber with High- Performance Microwave Absorption. Composites Part A 119: 1-7.

Nadgorny, M; Ameli, A (2018). Functional Polymers and Nanocomposites for 3D Printing of Smart Structures and Devices. Review-article. ACS Applied Materials \& Interfaces 10: 17489-17507.

Pang, Y; Cheng, H; Zhou, Y; Li, Z; Wang, J (2012). Ultrathin and Broadband High Impedance Surface Absorbers Based on Metamaterial Substrates. OPTICS EXPRESS 20 (11): 12515-12520.

Rahmanzadeh, M; Rajabalipanah, H; Abdolali, A (2018). Multilayer Graphene-Based Metasurfaces: Robust Design Method for Extremely Broadband, Wide-Angle, and Polarization-Insensitive Terahertz Absorbers. Appl. Opt. 57 (4): 959-968

Ren, J; Yin, J.Y (2018). 3D-Printed Low-Cost DielectricResonator-Based Ultra-Broadband Microwave Absorber Using Carbon-Loaded Acrylonitrile Butadiene Styrene Polymer. Materials 11: 1249.

Silva, M. W; Kretly, L. C (2011). A New Concept RAM-
Radiation Absorbent Material: Applying Corrugated Surfaces to Improve Reflectivity. In Microwave \& Optoelectronics Conference (IMOC).

Sood, D; Tripathi, C. C (2017). A Compact Ultrathin UltraWideband Metamaterial Microwave Absorber. Journal of Microwaves, Optoelectronics and Electromagnetic Applications 16 (2): 514-528.

Tao, H; Bingham, C. M; Strikwerda, A. C; Pilon, D; Shrekenhamer, D; Landy, N. I; Fan, K; Zhang, X; Padilla, W. J; Averitt, R. D (2008). Highly Flexible Wide Angle of Incidence Terahertz Metamaterial Absorber: Design, Fabrication , and Characterization. Physical Review B 78 (4): 241103-1 to 241103-4.

Tiep, D. H; Khuyen, B. X; Tung, B. S ; Kim, Y. J; Hwang, J. S; Lam, V. D; Lee, Y. P (2018). EnhancedBandwidth Perfect Absorption Based on a Hybrid Metamaterial. Optical Materials Express 8 (9): 27512759 .

Wang, B. X; Wang, L; Wang, G; Huang, W; Li, X; Zhai, X (2014). Theoretical Investigation of Broadband and Wide-Angle Terahertz Metamaterial Absorber. IEEE Photonics Technology Letters 26 (2): 111-114.

Wu, H; Xing, L; Cai, Y; Liu, L; He, E; Li, B; Tian, X (2020). A Study on the Fused Deposition Modeling Process of Graphene/Nano- $\mathrm{Fe}_{3} \mathrm{O}_{4}$ Composite Absorber and its Absorbing Properties of Electromagnetic Microwave. Applied Sciences 10 (1508): 1-12.

Zhang, L; Zhou, P; Chen, H; Lu, H; Xie, J; Deng, L (2015). Ultra-Thin Wideband Magnetic-Type Metamaterial Absorber Based on LC Resonator at Low Frequencies. Applied Physics A: Materials Science and Processing 121 (1): 233-38

Zhou, D; Huang, X; Du, Z (2017). Analysis and Design of Multi-Layered Broadband Radar Absorbing Metamaterial Using 3D Printing Technology- Based Method." IEEE Antennas and Wireless Propagation Letters, 16: 133-136,

Zhou, Q; Yin, X; Ye, F; Liu, X; Cheng, L; Zhang, L (2017). A Novel Two-Layer Periodic Stepped Structure for Effective Broadband Radar Electromagnetic Absorption. Materials \& Design 123: 46-53.

Zhu, J; Sun, W; Ding, F; He, Q; Zhou, L (2014). UltraBroadband Terahertz Metamaterial Absorber. Applied Physics Letters 105 (2): 021102 